\title{
Low CD4 count and educational status predict abnormal cervical smears amongst HIV-positive women initiating antiretroviral therapy in South Africa
}

\section{Authors:}

Nondumiso Mthembu ${ }^{1}$ (1)

Jienchi Dorward ${ }^{1,2}$

Nivashnee Naicker ${ }^{1}$ (1)

Farzana Osman ${ }^{1}$ (D)

Siphesihle Gumede ${ }^{1}$ (1)

Yukteshwar Sookrajh ${ }^{3}$ ৫

Paul Drain $4,5,6$ (1)

Nigel Garrett ${ }^{1,7}$ —

\section{Affiliations:}

${ }^{1}$ Centre for the AIDS

Programme of Research in

South Africa (CAPRISA),

University of KwaZulu-Natal,

Durban, South Africa

${ }^{2}$ Nuffield Department of

Primary Care Health Sciences, University of Oxford, Oxford,

United Kingdom

${ }^{3}$ Prince Cyril Zulu Communicable Disease

Centre, eThekwini

Municipality, Durban,

South Africa

${ }^{4}$ Department of Global Health, Schools of Medicine and Public Health, University of Washington, Seattle,

United States

${ }^{5}$ Department of Medicine, School of Medicine, University of Washington, Seattle, United States

${ }^{6}$ Department of Epidemiology, School of Public Health, University of Washington, Seattle, United States

Read online:

Scan this $Q R$ code with your smart phone or mobile device to read online.

\section{Introduction}

Cervical cancer is common amongst human immunodeficiency virus (HIV)-positive women in low- and middle-income countries. In South Africa, more than 7500 cases are diagnosed annually and over $50 \%$ result in death, making cervical cancer the leading cause of cancer mortality. ${ }^{1}$ South Africa's high HIV prevalence contributes to this high burden, because HIV-positive women are more likely to have persistent human papilloma virus (HPV) infection and precancerous cervical changes. ${ }^{2}$ Cervical cancer is preventable either through HPV vaccination of girls before sexual debut, which was rolled out in South Africa from 2014, or screening and treatment of precancerous cervical lesions. South African guidelines recommend cervical screening for all HIV-positive women at HIV diagnosis and then every 3 years. ${ }^{1}$

Antiretroviral therapy (ART) causes immune reconstitution and may reduce the risk of cervical cancer amongst HIV-positive women by lowering HPV acquisition, increasing HPV clearance and slowing the progression to precancerous lesions. ${ }^{2}$ However, these effects may be diminished for women who initiate ART at low CD4 counts. ${ }^{3}$ Since 2016, when universal test and treat (UTT) was introduced in South Africa, women began initiating ART at CD4 counts $>500$ cells $/ \mathrm{mm}^{3}$ (early initiators) and may therefore be protected against precancerous cervical abnormalities and cancer. $^{3}$

In this study, we aimed to assess whether early initiators of ART had a lower risk of abnormal cervical smears when compared to late initiators (women with a CD4 $\leq 500$ cells $/ \mathrm{mm}^{3}$ ), after introduction of UTT in South Africa.

\section{Methods}

\section{Study design}

We performed a cross-sectional analysis at enrolment into the Simplifying HIV TREAtment and Monitoring (STREAM) study, a randomised trial assessing point-of-care viral load testing amongst people living with HIV receiving ART (NCT03066128). ${ }^{4,5}$

\section{Participants and setting}

The STREAM study was conducted at the Prince Cyril Zulu Communicable Disease Centre (PCZ CDC), a large public clinic in central Durban, South Africa. At PCZ CDC, all women who test HIV-positive are referred for ART initiation and have CD4 cell count and Papanicolaou cervical smear testing performed using routine National Health Laboratory Services (NHLS). We enrolled non-pregnant, HIV-positive adults aged 18 years or older who were clinically stable on first-line ART for 6 months, and randomised them to receive point-of-care viral load monitoring and taskshifting to an enrolled nurse or standard laboratory monitoring and professional nurse care. ${ }^{4}$

${ }^{7}$ Department of Public Health Medicine, School of Nursing and Public Health, University of KwaZulu-Natal, Durban, South Africa Corresponding author: Jienchi Dorward, jienchi.dorward@caprisa.org

Project Research Number: NCT03066128

Dates: Received: 18 Nov. 2019 | Accepted: 22 Jan. 2020 | Published: 30 Mar. 2020

How to cite this article: Mthembu N, Dorward J, Naicker N, et al. Low CD4 count and educational status predict abnormal cervical smears amongst HIV-positive women initiating antiretroviral therapy in South Africa. S Afr J HIV Med. 2020;21(1), a1045. https://doi.org/10.4102/ sajhivmed.v21i1.1045

Copyright: (C 2020. The Authors. Licensee: AOSIS. This work is licensed under the Creative Commons Attribution License. 
All participants had been initiated on ART after the introduction of UTT. Cervical smear results were checked using NHLS records for all women at screening. Women with abnormal cervical smear results of high-grade squamous intraepithelial lesion (HSIL) who needed referral for colposcopy did not meet the STREAM study eligibility criteria of being clinically stable (as they required active care by a physician) and were excluded from STREAM, and therefore from this analysis. ${ }^{4}$ Those without a baseline cervical smear result had a smear test after entry into the study and continued follow-up, even if a high-grade lesion was detected.

\section{Data source and data management}

At enrolment, we recorded socio-demographic, laboratory and clinical information using a nurse-administered questionnaire and by retrospective review of clinical notes and NHLS records. We used the following validated questionnaires: Audit- $\mathrm{C}$ to assess alcohol use, Patient Health Questionnaire 2 (PHQ2) for depression and World Health Organization Violence Against Women for intimate partner violence. We captured data in the secure, password-protected iDataFax system (DF/Net Research, Inc., Seattle, WA, USA) and performed three rounds of data quality checks consisting of source document review, internal quality audits and weekly quality reports.

\section{Statistical analysis}

We assessed socio-demographic and clinical exposure variables including age, educational status, having a stable partner, parity, contraceptive use, time from diagnosis to ART initiation, previous tuberculosis, intimate partner violence, alcohol use or depression and CD4 count. The primary outcome for this analysis was the absence or the presence of an abnormal cervical smear, which included atypical squamous cells of undetermined significance (ASCUS), low-grade squamous intraepithelial lesion (LSIL) and HSIL. We used descriptive statistics to summarise socio-demographic and clinical variables and missing data. Associations between socio-demographic and clinical exposures, and the outcome of an abnormal cervical smear result were assessed using bivariable and multivariable Poisson regression models with robust variance. We included covariates with a $p$-value of $<0.15$ in the bivariable analysis into the multivariable model. We used SAS version 9.4 (SAS Institute Inc., Cary, NC, USA) for the statistical analysis.

\section{Results}

A total of 390 participants were enrolled into the STREAM study, of whom $235(60.3 \%)$ were women, with a median age of 30 years (interquartile range [IQR] 26-37 years) and a median CD4 count of $401 /$ cells $/ \mathrm{mm}^{3}$ (IQR 215-608 cells $/ \mathrm{mm}^{3}$ ). Eight women who had HSIL at ART initiation were not eligible for enrolment and thus were excluded from the STREAM study. Of the 235 women, $176(74.9 \%)$ women with a conclusive cervical smear result were included in this analysis (Table 1), and of the 176 women included, 66 (37.5\%) had abnormal cervical smears. Amongst the women with abnormal cervical smears, 62 women (35.2\%) had LSIL, three (1.7\%) had HSIL and one $(0.6 \%)$ had ASCUS. Women with a CD4 count $<200$ cells $/ \mathrm{mm}^{3}$ had the highest proportion of abnormal cervical smears $(20 / 40,50.0 \%)$. Of the 59 women with no cervical smear test result available, eight $(13.6 \%)$ tests were performed but no test result was available, $13(22.0 \%)$ were inadequate for evaluation and $38(64.4 \%)$ had no record of testing. Median CD4 count amongst women with no smear result was 405 (IQR 244-608), compared to 401 (IQR 207-611) amongst those with a smear result $(p=0.498)$.

In bivariable analysis, women with an initiation CD4 count of $\leq 500$ cells $/ \mathrm{mm}^{3}$ had a higher risk of abnormal cervical smears, compared to women with a CD4 count $>500$ cells $/ \mathrm{mm}^{3}$ (risk ratio 1.84, 95\% confidence interval [CI] 1.04-3.28, Table 2). Women who had not completed secondary education also had a higher risk of abnormal cervical smears. Time from diagnosis to ART initiation, having a stable partner, previous tuberculosis, contraceptive use, intimate partner violence, alcohol use or depression were not associated with abnormal cervical smear results.

In multivariable analyses, late initiators of ART had a higher risk of abnormal cervical smears when compared to early initiators (adjusted risk ratio [aRR] 1.71, 95\% CI 1.04-2.80). Furthermore, independent of CD4 count, women with a lower educational status also had a higher risk of abnormal cervical smears (aRR 1.48, 95\% Cl 1.02-2.14).

TABLE 1: Baseline characteristics of women with cervical smear results available $(N=176) .^{5}$

\begin{tabular}{|c|c|c|c|c|}
\hline \multirow[t]{2}{*}{ Characteristics } & \multirow[t]{2}{*}{ Level } & \multicolumn{3}{|c|}{ Overall } \\
\hline & & $\%$ & $n$ & $N$ \\
\hline \multirow[t]{3}{*}{ Age } & $\leq 24$ & 21.0 & 37 & 176 \\
\hline & $25-34$ & 44.9 & 79 & 176 \\
\hline & $\geq 35$ & 34.1 & 60 & 176 \\
\hline \multirow[t]{2}{*}{ Secondary education } & Yes & 55.4 & 97 & 175 \\
\hline & No & 44.6 & 78 & 175 \\
\hline \multirow[t]{2}{*}{ Regular or stable partner } & Yes & 78.4 & 138 & 176 \\
\hline & No & 21.6 & 38 & 176 \\
\hline \multirow[t]{2}{*}{ Time from diagnosis to initiation } & $\leq 6$ months & 67.1 & 112 & 167 \\
\hline & $>6$ months & 32.9 & 55 & 167 \\
\hline \multirow[t]{2}{*}{ Previous history of tuberculosis } & Yes & 11.4 & 20 & 176 \\
\hline & No & 88.6 & 156 & 176 \\
\hline \multirow[t]{2}{*}{ Risky alcohol intake $\dagger$} & Yes & 19.4 & 28 & 144 \\
\hline & No & 80.6 & 116 & 144 \\
\hline \multirow{2}{*}{$\begin{array}{l}\text { Positive depression screen (PHQ- } \\
2 \text { score of } 2 \text { or more) }\end{array}$} & Yes & 8.5 & 15 & 176 \\
\hline & No & 91.5 & 161 & 176 \\
\hline \multirow{2}{*}{$\begin{array}{l}\text { Any history of intimate partner } \\
\text { violence }\end{array}$} & Yes & 17.7 & 31 & 175 \\
\hline & No & 82.3 & 144 & 175 \\
\hline \multirow[t]{2}{*}{ CD4 count (cells $/ \mathrm{mm}^{3}$ ) } & $\leq 500$ & 35.2 & 62 & 176 \\
\hline & $>500$ & 64.8 & 114 & 176 \\
\hline \multirow[t]{2}{*}{ Children } & None & 19.3 & 34 & 176 \\
\hline & 1 or more & 80.7 & 142 & 176 \\
\hline \multirow[t]{2}{*}{ Contraception use } & Yes & 14.2 & 25 & 176 \\
\hline & No & 85.8 & 151 & 176 \\
\hline
\end{tabular}

Source: Drain PK, Dorward J, Violette LR, et al. Point-of-care HIV viral load testing combined with task shifting to improve treatment outcomes (STREAM): Findings from an open-label, non-inferiority, randomised controlled trial. Lancet HIV. 2020. https://doi.org/10.1016/ S2352-3018(19)30402-3. Epub ahead of print.

PHQ-2, Patient Health Questionnaire 2.

$\dagger$, In women, an AUDIT-C score of 3 or more is considered positive for at-risk alcohol use. 
TABLE 2: Associations between socio-demographic variables, initiation CD4 count and abnormal cervical smear results $(n=176) .^{5}$

\begin{tabular}{|c|c|c|c|c|c|c|c|c|c|c|}
\hline \multirow[t]{2}{*}{ Characteristic } & \multirow[t]{2}{*}{ Level } & \multicolumn{3}{|c|}{ Abnormal cervical smear } & \multicolumn{2}{|c|}{ Risk ratio } & \multirow[t]{2}{*}{$p$} & \multicolumn{2}{|c|}{ Adjusted risk ratio } & \multirow[t]{2}{*}{$p$} \\
\hline & & $n$ & $N$ & $\%$ & $95 \%$ & $\mathrm{Cl}$ & & $95 \%$ & $\mathrm{Cl}$ & \\
\hline \multirow[t]{2}{*}{ Initiation CD4 count (cells/mm³) } & $>500$ & 15 & 62 & 24.2 & 1 & - & - & 1 & - & - \\
\hline & $\leq 500$ & 51 & 114 & 44.7 & 1.84 & $1.04-3.28$ & 0.037 & 1.71 & $1.04-2.80$ & 0.034 \\
\hline \multirow[t]{3}{*}{ Age (years) } & $18-24$ & 10 & 37 & 27.0 & 1 & - & - & 1 & - & - \\
\hline & $25-34$ & 37 & 79 & 46.8 & 1.72 & $0.86-3.47$ & 0.126 & 1.52 & $0.77-3.00$ & 0.233 \\
\hline & $\geq 35$ & 19 & 60 & 31.7 & 1.17 & $0.55-2.52$ & 0.682 & 1.01 & $0.49-2.05$ & 0.989 \\
\hline & No & 37 & 78 & 47.4 & 1.58 & $0.97-2.57$ & 0.066 & 1.48 & $1.02-2.14$ & 0.038 \\
\hline \multirow[t]{2}{*}{ Number of children } & 0 & 8 & 34 & 23.5 & 1 & - & - & 1 & - & - \\
\hline & $\geq 1$ & 58 & 142 & 40.8 & 1.76 & $0.84-3.68$ & 0.136 & 1.5 & $0.70-3.24$ & 0.297 \\
\hline \multirow{2}{*}{$\begin{array}{l}\text { Time from diagnosis to initiation } \\
>6 \text { months? }\end{array}$} & No & 43 & 112 & 38.4 & 1 & - & - & - & - & - \\
\hline & Yes & 18 & 55 & 32.7 & 0.86 & $0.50-1.49$ & 0.594 & - & - & - \\
\hline \multirow[t]{2}{*}{ Regular or stable partner } & No & 13 & 38 & 34.2 & 1 & - & - & - & - & - \\
\hline & Yes & 53 & 138 & 38.4 & 1.13 & $0.61-2.07$ & 0.699 & - & - & - \\
\hline & Yes & 10 & 20 & 50.0 & 1.41 & $0.71-2.77$ & 0.317 & - & - & - \\
\hline \multirow[t]{2}{*}{ Contraception use } & No & 54 & 151 & 35.8 & 1 & - & - & - & - & - \\
\hline & Yes & 12 & 25 & 48.0 & 1.31 & $0.68-2.49$ & 0.415 & - & - & - \\
\hline \multirow[t]{2}{*}{ History of intimate partner violence } & No & 54 & 144 & 37.5 & 1 & - & - & - & - & - \\
\hline & Yes & 12 & 31 & 38.7 & 1.03 & $0.55-1.93$ & 0.917 & - & - & - \\
\hline \multirow[t]{2}{*}{ Risky alcohol intake† } & No & 43 & 116 & 37.1 & 1 & - & - & - & - & - \\
\hline & Yes & 7 & 28 & 25.0 & 0.67 & $0.30-1.50$ & 0.334 & - & - & - \\
\hline \multirow{2}{*}{$\begin{array}{l}\text { Positive depression screen (PHQ-2 } \\
\text { score } \geq 2 \text { ) }\end{array}$} & No & 59 & 161 & 36.6 & 1 & - & - & - & - & - \\
\hline & Yes & 7 & 15 & 46.7 & 1.25 & $0.57-2.75$ & 0.577 & - & - & - \\
\hline
\end{tabular}

Source: Drain PK, Dorward J, Violette LR, et al. Point-of-care HIV viral load testing combined with task shifting to improve treatment outcomes (STREAM): Findings from an open-label, noninferiority, randomised controlled trial. Lancet HIV. 2020. https://doi.org/10.1016/S2352-3018(19)30402-3. Epub ahead of print.

$\mathrm{Cl}$, confidence interval; PHQ-2, Patient Health Questionnaire 2.

$\dagger$, In women, an AUDIT-C score of 3 or more is considered positive for at-risk alcohol use.

\section{Discussion}

In this cohort of women initiating ART after implementation of UTT, there was a high prevalence of abnormal cervical lesions, affecting over a third of all women successfully screened. Women who initiated ART at lower CD4 thresholds and those who did not reach secondary education had a higher risk for abnormal cervical lesions.

Our findings are similar to a systematic review, including studies mainly from Europe and North America, which found that women with lower CD4 counts had consistently higher incidence of abnormal cervical lesions. ${ }^{6}$ This review also showed an increased risk of progression of cervical lesions with declining CD4 count. ${ }^{6}$ A cross-sectional analysis of 1140 Nigerian women showed that both low- and high-grade cervical lesions were detected almost four times more frequently in women with CD4 $<200$ cells $/ \mathrm{mm}^{3}{ }^{3}{ }^{7}$ Therefore, earlier initiation of ART at higher CD4 counts may have a protective effect on the development and progression of abnormal cervical lesions in this setting. ${ }^{2,3,8}$ Of note, in spite of the implementation of UTT, the majority of women in this study presented with CD4 counts $\leq 500$ cells $/ \mathrm{mm}^{3}$, meaning that further efforts are needed to diagnose and initiate ART earlier amongst women living with HIV.

Similar to our finding, a cross-sectional study from Zambia, analysing data in over 14000 women from a National Cervical Screening Programme, showed that women having at least secondary education were less likely to develop abnormal cervical lesions, compared to women with no formal education. ${ }^{9}$ The protective effect of attaining a higher level of education may be explained by increased awareness of the disease, delayed sexual debut and greater access to healthcare, including cervical screening services. While the recent roll-out of HPV vaccination to South African school girls through a public health initiative has somewhat created renewed awareness of cervical cancer, gaps remain in knowledge and prevention of the disease and access to screening services for secondary prevention. ${ }^{1,10}$

Limitations of our study included the cross-sectional design and a relatively small sample size, which means that the analysis may not be sufficiently powered to rule out weak associations between other exposure variables and abnormal smears. Furthermore, we excluded women with HSIL without a conclusive negative colposcopy result from the study. Even in this large HIV clinic, a quarter of women did not have the recommended cervical smear result available at ART initiation. A strength of our study is that we were able to assess the prevalence of abnormal cervical lesions in women initiating ART at higher CD4 thresholds in the era of UTT in a public health setting.

While HPV vaccination for young women has now been introduced in South Africa, the long-term impact on cervical cancer is still uncertain. In the meantime, cervical cancer screening remains a priority. Here, we highlight that early ART initiation and ensuring frequent cervical screening for those with lower CD4 counts and lower educational background should be prioritised. Universal test and 
treat will help reduce cervical abnormalities, but other measures, such as scaling up of existing screening services, better colposcopy and treatment provision, as well as the implementation of high-risk HPV screening strategies, are necessary to reduce the burden of this preventable disease.

\section{Acknowledgements}

The authors are grateful to the study participants and the staff at the Prince Cyril Zulu Communicable Disease Centre and Centre for the AIDS Programme of Research in South Africa (CAPRISA) eThekwini Clinical Research Site.

\section{Competing interests}

The authors report no real or perceived vested interests related to this article that could be construed as a conflict of interest.

\section{Ethical consideration}

Ethical approval to conduct the study was obtained by the University of KwaZulu-Natal Biomedical Research Ethics Committee (BFC296/16) and the University of Washington Institutional Review Board (STUDY00001466).

\section{Authors' contributions}

N.M., J.D., N.N., P.D. and N.G. conceived the study. N.M., J.D., N.N., S.G. and Y.S. collected the data. F.O. performed the statistical analysis. N.M. and J.D. drafted the manuscript. All authors critically reviewed and edited the manuscript and gave their consent for publication.

\section{Funding information}

The Simplifying HIV TREAtment and Monitoring (STREAM) study was funded by the US National Institutes for Health (NIH) (AI124719). The NIH had no role in the study design and manuscript submission, or collection, management, analysis or interpretation of study data.

\section{Data availability statement}

The data that support the findings of this study are available from the corresponding author (J.D.) upon reasonable request and subject to CAPRISA data availability policies.

\section{Disclaimer}

The views and opinions expressed in this article are those of the authors and do not necessarily reflect the official policy or position of any affiliated agency of the authors.

\section{References}

1. The South African National Department of Health. Cervical cancer prevention and control [homepage on the Internet]. Pretoria; 2017 [cited 21 Jan 2020]. Available from: http://www.health.gov.za/index.php/2014-08-15-12-53-24?download=1393. cervical-cancer-policy-pdf

2. Liu G, Sharma M, Tan N, et al. HIV-positive women have higher risk of human papilloma virus infection, precancerous lesions, and cervical cancer. AIDS. 2018;32(6):795-808. https://doi.org/10.1097/QAD.0000000000001765

3. Kelly $H$, Weiss $H A$, Benavente $Y$, et al. Association of antiretroviral therapy with high-risk human papillomavirus, cervical intraepithelial neoplasia, and invasive cervical cancer in women living with HIV: A systematic review and meta-analysis. Lancet HIV. 2018;5(1):e45-e58. https://doi.org/10.1016/S2352 3018(17)30149-2

4. Dorward J, Garrett N, Quame-Amaglo J, et al. Protocol for a randomised controlled implementation trial of point-of-care viral load testing and task shifting: The Simplifying HIV TREAtment and Monitoring (STREAM) study. BMJ Open. 2017;7(9):e017507. https://doi.org/10.1136/bmjopen-2017-017507

5. Drain PK, Dorward J, Violette LR, et al. Point-of-care HIV viral load testing combined with task shifting to improve treatment outcomes (STREAM): Findings from an open-label, non-inferiority, randomised controlled trial. Lancet HIV. 2020 https://doi.org/10.1016/S2352-3018(19)30402-3. Epub ahead of print.

6. Denslow $\mathrm{SA}$, Rositch $\mathrm{AF}$, Firnhaber $\mathrm{C}$, et al. Incidence and progression of cervical lesions in women with HIV: A systematic global review. Int J STD AIDS. 2014;25(3):163-177. https://doi.org/10.1177/0956462413491735

7. Ezechi OC, Pettersson KO, Okolo CA, et al. The association between HIV infection, antiretroviral therapy and cervical squamous intraepithelial lesions in South Western Nigerian women. PLoS One. 2014;9(5):e97150. https://doi.org/10.1371/ journal.pone.0097150

8. Kelly HA, Sawadogo B, Chikandiwa A, et al. Epidemiology of high-risk human papillomavirus and cervical lesions in African women living with HIV/AIDS. AIDS. 2017;31(2):273-285. https://doi.org/10.1097/QAD.0000000000001301

9. Hamoonga TE, Likwa RN, Musonda $\mathrm{P}$, et al. Higher educational attainment associated with reduced likelihood of abnormal cervical lesions among Zambian women - A cross sectional study. BMC Cancer. 2017;17(1):681. https://doi. org/10.1186/s12885-017-3680-z

10. Ramathuba DU, Ngambi D. Knowledge and attitudes of women towards human papilloma virus and HPV vaccine in thulamela municipality of vhembe district in Limpopo province, South Africa. Afr J Reprod Health. 2018;22(3):111-119. https://doi.org/10.29063/ajrh2018/v22i3.12 